\title{
Systems Engineering as the Basis for Design Collaboration
}

\author{
Eva Russwurm*, Florian Faltus, Joerg Franke \\ Chair of Factory Automation and Production Systems, Friedrich-Alexander Universität Erlangen-Nürnberg, \\ Egerlandstraße 7-9, 91058 Erlangen, Germany; *eva.russwurm@faps.fau.de
}

SNE 31(4), 2021, 201-208, DOI: 10.11128/sne.31.tn.10583 Received: 2021-09-10 (Selected ASIM WS 2021 Postconf. Pub., English version); Revised: 2021-11-19; Accepted: 2021-11-28 SNE - Simulation Notes Europe, ARGESIM Publisher Vienna ISSN Print 2305-9974, Online 2306-0271, www.sne-journal.org

Abstract. Systems engineering (SE) approach is undergoing constant change and is already being used in many companies as part of product development. This approach, which offers many advantages in collaboration, efficiency and product quality, as well as cost, can meet the challenges of Industry 4.0. Various tools are needed to implement SE. These include a PLM system for collaboration during development, as well as various simulation environments that are brought together to form a co-simulation. One way to enable communication for this is to use the OPC UA communication standard. The application of the SE approach will be illustrated using two application examples, namely a holistic simulation of a production plant with energy management and a teaching course.

\section{Introduction}

The automation of production systems is characterized by high technical complexity and strong interdisciplinarity. The planning of an industrial plant requires the coordination and integration of various specialist disciplines such as mechanical, process and electrical engineering or software with regard to the procedure and work results.

In this context, automation as a connector ensures the correct interaction of various disciplines. The Industry 4.0 approach and the associated increase in IT penetration in the manufacturing industry are significantly increasing the relevance of digitalization in production.

This is already evident during product development, because engineers from different disciplines already work together as part of systems engineering. This requires a data platform that ensures data consistency and enables access, as well as the possibility of exchange through all disciplines.
This article first gives an overview of the methodology of continous engineering and classifies the development methodology according to the V-model. It will then be shown how the individual disciplines can participate synchronously in a simulation and thus how virtual commissioning can succeed.

Finally, the application of integrated engineering will be presented based on two use cases; on the one hand, the methodical approach at university with students as a basis for future collaboration in the company and, on the other hand, the development of a co-simulation for an automated production plant in an industrial company.

\section{Continuous Engineering}

In modern mechanical engineering, there are hardly any purely mechanical products anymore. The share of electronics and software or machine control in the product development process (PDP) is not only continuously increasing, see Figurure 1, but is also becoming more and more complex.

Whereas in the 1980s an industrial plant consisted mainly of mechanics and mechatronics was more of a supporting accessory, today it is the software that decisively determines the functionalities of a plant. The reasons for this arise from customer requirements such as process flexibilization and networking of all plants in operation to enable simple operation and monitoring of production [1].

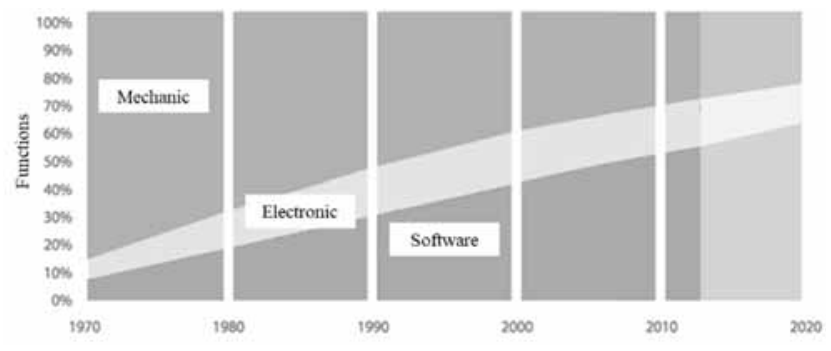

Figure 1: Proportion of individual disciplines in the PEP [1]. 
Therefore, successful product development already requires successful interaction between various fields of expertise: mechanical engineering, electrical engineering and computer science, especially software engineering. This complexity can no longer be covered by a small number of people, so development teams have to be more interdisciplinary and larger than they were in the past. This inevitably means that the groups also have to work together in different locations in different time zones.

\subsection{Definition of Mechatronic Systems}

The term "mechatronics" is an artificial word composed of mechanics and electronics and refers to "the synergetic interaction of the disciplines of mechanical engineering, electrical engineering and information technology in the design and manufacture of industrial products, as well as in process design" [2]. The interdisciplinary interaction of a high number of coupled elements, interfaces and interactions of the mechatronic modules with each other makes the application of a holistic, cross-disciplinary approach to system definition indispensable. This also includes communication and cooperation between the individual disciplines [3].

For this reason, new development methods based on the fundamental idea of systems engineering (SE) are increasingly being used for mechatronic systems.

\subsection{Strategy of Continous Engineering}

The three guiding principles of simultaneous engineering are parallelization, standardization and integration. Parallelization means optimizing the timing of sub-processes that are independent of each other and can be processed independently. In standardization, the primary goal is to avoid duplication and repetition of work. This is achieved primarily through a specified uniform design of modules, components, phases and interfaces between projects and departments. The goal of integration is to turn interfaces into seams. They allow all product information to be brought together.

\subsection{Multidisciplinary Approach and Parallelization of Development Activities}

This multidisciplinary structure of the model is based on an iterative procedure. Integration progress and synchronization points are continuously checked and guarantee successful product development due to early error prevention.
Furthermore, parallelization instead of sequential product development can save valuable time, which has a positive effect on the required time target (time-to-market). In order to meet this target, certain methods, such as reuse within the SE, must be applied [4].

Consequently, the use of SE to support the upcoming transformation to customized products should be considered as a possible solution approach.

\section{Product Development along the V-model}

\subsection{Requirement and Solution Specification}

Another concept, which is mainly used in the requirement engineering, i.e. the development of requirements from abstract specifications of the customer, is the method of RFLP [5]. In German-language literature also named AFLP, it describes the subdivision into requirements, functions, logic and physical model. By these four ranges the goal of the concept, i.e. the draft of a uniform structure, which can be applied to all disciplines, can be pursued.

This interdisciplinary combination results in an initially discipline-independent description of the system and takes on an extraordinary role especially in the early development phases of multidisciplinary products.

The physical model describes the elaboration of the system architecture by adding physical properties using discipline-specific methods and IT applications [6].

\subsection{Mechanical, Electrical and Software Development}

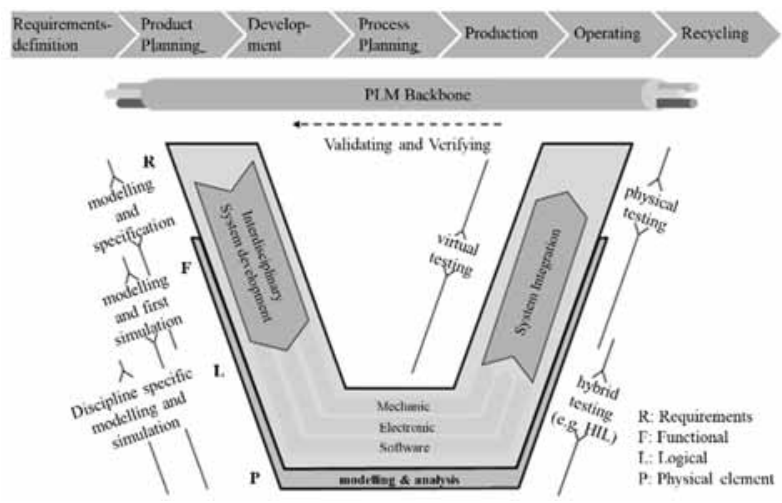

Figure 2: MVPE process model based on VDI 2206 [3]. 
In systems engineering process, mechanical design follows the solution specification. Simultaneously with it, the electrical design and the software development are proceeded, as can be seen in Figure 2. After completion of the design phase, to which it belongs, realization follows, as well as virtual and actual commissioning [3].

In order to enable a continuous flow of information and a coherent product model across disciplines, the respective discipline naturally has interfaces with other disciplines (mechanical, electrical and software). For this purpose, specifically new approaches of product development have to be defined [7]. The interfaces are predominantly mapped by the PLM system.

\subsection{Further Development to Model-based Systems Engineering}

One possible approach is Model-Based Systems Engineering (MBSE), which describes the transition from document-based to model-based systems engineering and combines MVPE with systems engineering. It is a "formalized application of modeling to support the incorporation of system requirements, design, analysis, verification, and validation from the concept phase through the development phase to later life cycle phases" [8]. In this case, the draft engineering is of particular importance, since it is during this phase that the system model is created, which contains all the product lifecycle requirements of the product.

Completely digital product models (plant models) are already created during the product development process, enabling physical products to be linked to the associated virtual models from product development. This enables the networking of real production facilities with the digital images created during product planning, as required by Industry 4.0 [10], which helps to merge the virtual and real world.

Within the framework of MBSE, a holistic and consistent data model is created for each product over the entire product lifecycle, which enables enormous productivity, efficiency and quality increases as real and digital processes overlap. For example, in addition to the product, manufacturing is also planned digitally and mapped and validated through early simulations, resulting in up to $50 \%$ reduction in time-to-market for new products [11]. The end-to-end data model enables flexibility in the production process through consistency, which allows products to be individualized.

\subsection{Integration of MBSE into the Stage-Gate Process and the Quality Gate Model}

The VDMA Quality Gate model corresponds to a stagegate process based on Cooper's model [9] . It divides innovation and product development processes into different stages, to which similar activities are grouped. The individual stages end with a quality control (gate), which can only be crossed if the defined requirements are filfilled.

The decisive factor is the assessment of the management. One advantage of this method can be found in the structuring of long processes, which become thereby controllable and steerable. The regular reconciliation of the project team with management also serves to inform all stakeholders and involves interdisciplinary company departments (marketing, sales, etc.) early in the process. Overall, stage-gate processes lead to higher quality and more innovations [9], [12].

The procedure has many parallels to the V-Modell of the VDI standard 2206 and both models comprise almost the same steps. The description of the phase contents, deliverables and gate criteria turn the general V-Modell into a controllable process. However, the work here is not yet model-based, because the greatest challenge is the realization of consistency in the PDP by means of consistent system models.

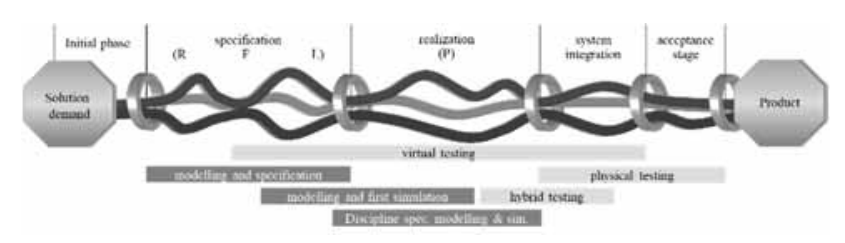

Figure 1: Model-based quality gate approach.

Figure 3 illustrates the modified quality gate approach. As in the VDMA quality gate model, the turquoise, yellow and red bands represent the classic disciplines of mechanics, electronics and software, which continue to be processed as parallel strands [13], [14].

During further development, the requirements and solution specification phases are combined into a single specification phase so that a holistic system model consisting of system requirements, functions and logic (R, F, L) can be created and released. The creation of test specifications also forms part of this phase. The performance of quantitative simulations also supports the objective selection of solution alternatives. 
The subsequent realization phase includes disciplinespecific modeling and simulation, which is combined in the integration phase at the latest and supplemented by physical tests. Final tests are performed in the acceptance phase.

During the PEP, when working with evolving models, simulation is of great importance as a supporting module. Furthermore, the first simulation studies, which, as shown in Figure 1ure 3, already start in the specification phase, form the basis for the digital twin of the product or the production plant [15]

\section{Co-simulation for Synchroni- zation of the Disciplines}

"Simulation is the recreation of a system with its dynamic processes in a model capable of experimentation in order to arrive at knowledge that can be applied to reality" [16, p. 3].

\subsection{Production System Simulation}

Nowadays, it is necessary to design an automated production system not only in terms of strength in mechanics (e.g. by means of finite element simulation), but to consider the entire mechatronic model and to take into account interactions between modules and models, because it is a complex system. This complexity increases with the number of elements as well as with the links between the elements in terms of tolerance or dynamics.

In order to make the complexity manageable, the system is divided into several - partly hierarchical - levels. In each level, relevant questions can be answered by the simulation.

The first step is to consider, evaluate and, if necessary, improve the issues and solutions in the individual levels. This is performed independently from simulations of other levels.

In context of MBSE, this means that individual components of the plant can be considered separately, but at the same time that all individual disciplines can be considered independently of each other. The fact that the second approach will not result in meaningful solutions is proven at the latest when considering mechatronic models, because mechanical, electrical and information technology (software) tasks must be solved in an integrated manner.
Overall, simulation has proven to be a profitable method to highlight discussion points, but also to support complex decision-making processes [17]. Thus, simulation technology offers great opportunities for improvement in planning and operation of production systems [18].

\subsection{Multiphysical Simulation Programs}

There are already simulation programs from various manufacturers that are predestined for solving mechatronic requirements. Examples include the Mechatronics Concept Designer (MCD) from Siemens PLM, iPhysics from machineering or virtuos from isg. These systems not only offer a simulation environment, but also interfaces for virtual commissioning. [19].

These are tools for mechanical and electromechanical simulation (computer-aided engineering), manufacturing, tool and fixture design, quality inspection, and mechatronic concept development [20]. In this context, geometries modeled in mechanical design can be extended to a simulation model which, in addition to multi-body physics-based simulation, also includes aspects of automation technology and thus visualizes the physical behavior of different solution concepts. Based on the MBSE approach and a cross-domain solution concept, the simulation tools promote early interdisciplinary collaboration between mechanics, electronics and software development. This is reflected in particular by cost savings and accelerated product development times.

\subsection{Coupling of Simulation Programs}

The aforementioned increasing degree of complexity of production systems makes it necessary to link additional simulation environments. To this end, distributed simulation has gained in importance in recent years. Initially, this involves a simulation model that is divided into different models in the sense of different levels. Data flows exist between the individual submodels via databases, which ensure their consistency. Digital product data in the form of work plans, calculations and CAD models are available in various database systems and can be used and modified by all those involved in the development process. The resulting product or product data models, which function both as interdisciplinary information carriers and as a link between the individual product development areas, such as planning and design, form the basis of distributed simulation. 


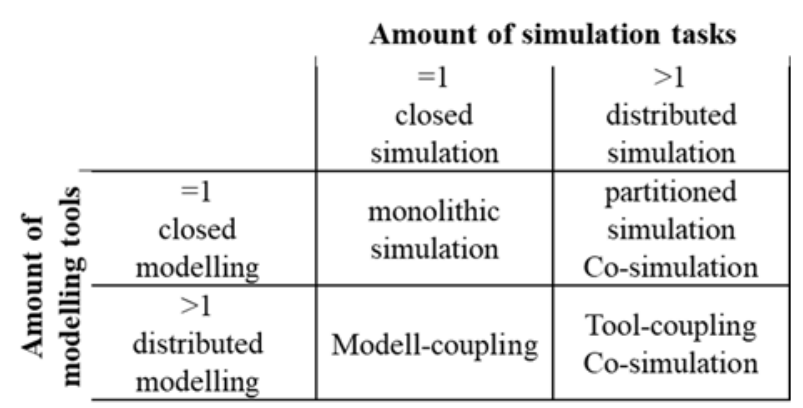

Table 1: Matrix of simulation architectures -[21].

Distributed simulation is also referred to as co-simulation [21], and can consist of a partitioned simulation or a tool coupling, as shown in Table 1.

The aforementioned discipline-specific simulation solutions developed in the context of the digital factory are very powerful in terms of mapping accuracy and calculation performance. However, these are designed for the virtual mapping of selected processes.

For a comprehensive modeling of the entire process, machine and plant behavior with all occurring interactions, high-resolution submodels of various simulation domains must be integrated into the digital image of the production plant and coupled in an overall simulation [22]

In the context of multidisciplinary modeling of $\mathrm{NC}$ machine tools, a central problem in unifying models from different simulation disciplines into an overall multidisciplinary model is said to challenging [23]. This is because, in principle, the relevant data of the individual models must be provided via a neutral uniform data interface by means of a uniform data format. Manufacturers of simulation software meanwhile confirm this thesis by integrating neutral data interfaces [24] .

Furthermore, a simulation tool is needed as a basis that passes on the data of all individual simulation tools. For this purpose, there are different approaches and tests in various contexts, but no system that has become established. In the use cases shown here, an approach for a cosimulation for the holistic simulation of a production plant operated with direct current is to be demonstrated.

\section{IT Infrastructure}

A functional and far-reaching infrastructure is required for the implementation of MBSE and the associated simulation. PLM systems are a common platform for the sensible implementation of SE in order to support and map model-based work.
For the mapping of co-simulations, additional data exchange formats are required that are as real-time-capable as possible.

\subsection{PLM System as the Basis for MBSE}

Product Lifecycle Management (PLM) describes a "holistic approach to enterprise-wide management and control of all product data and processes throughout the lifecycle along the extended logistics chain" [25].

As part of the change in development methodology, the engineer's tasks have changed from creative development activity to organizational and communication tasks (planning, procurement). Corresponding IT tools for more efficient management of the tasks are necessary [26] in order to master digitization.

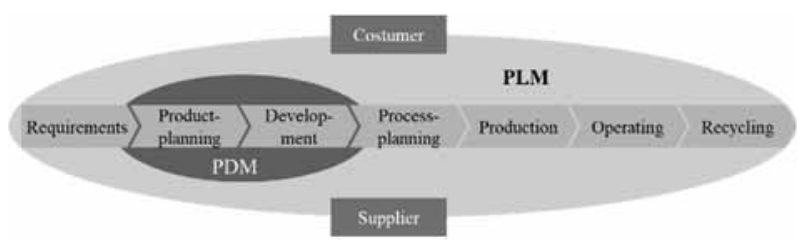

Figure 4: Distinction between PLM and PDM according to [27].

These challenges can only be overcome through the use of product data management (PDM) and PLM software [27]. The former focus on the product development phase, while the PLM approach expands the concept by considering the entire life cycle from initial idea to recycling [28]. Accordingly, PLM represents a concept rather than an IT system.

The PLM software is a component of the concept, serves to integrate IT tools into a development environment and supports the interaction of the tools in terms of models, systems, processes and procedures [3]. Holistic PLM concepts result from strategy, processes and the IT solution [32].

\subsection{OPC UA as Communication Standard}

In the field of automation, the goal of developing more effectively, more cost-effectively and more time-efficiently and, if possible in the sense of "first-time-right", to commission plants directly at the customer's site ready for production, is at the top of the list.

Just as the need for standardization and modularization in the software landscape is becoming louder, there is an increased demand for a standardized interface between several components at the communication level. 
Regarding this, OPC has emerged. OPC stands for "Open Platform Communications" and is a communication protocol that is used primarily in the context of Industrie 4.0 and that enables standardized access to devices, machines and other systems in the industrial environment. It forms the interface between control systems and the control level, enabling uniform data exchange regardless of the manufacturer. The most current specification is "Unified Architecture" or UA for short. OPC UA consists of a server and the client, with the OPC UA server forming the basis. The logical counterpart to the OPC UA server is the client. By connecting to the server, the data provided by the server can be read out [29], [30].

With the help of the described OPC UA standard, the co-simulation for an automated production plant was realized, which is supplied with its own industrial-level DC circuit with a voltage of $650 \mathrm{~V} \mathrm{DC}$.

Here, in addition to the multiphysical simulation of the process flow, the simulation of the power supply network plays a significant role.

\section{Virtual Commissioning as System Integration}

Virtual commissioning is a tool of the digital factory. It is assigned to the "planning of production facilities" phase and thus takes place before the "assembly and commissioning of production facilities" phase [31]. It describes the control commissioning on a virtual machine model, which represents the mechanical, electrical, pneumatic and hydraulic functionalities of an automated, mechatronic plant [33].

In the three types of virtual commissioning (modelin-the-loop (Mil), software-in-the-loop (Sil) and hardware-in-the-loop (Hil)), testing is always performed on the model. The aim is therefore to model the model as realistically as possible. The terms "Model", "Software" and "Hardware" in this context refer to the form of the control that is used, the control within the model, control by a simulated Programmable Logic Controller (PLC) (via the program PLCSim Advanced) or the control as a hardware component.

At any early stage, simulation can be used according to model-in-the-loop. Here, the sequence control is inserted directly into the physical model and tested within the model. This is more of a process-accompanying simulation, since individual steps are successively taken over by the control system.
At this point, however, the physical plant model is already used, which is also used in the other two forms of virtual commissioning. In a further step, the control program can first be tested with a simulated control on the model (Sil). Compared to Hil, this has the advantage that the entire periphery (input and output modules) does not yet have to be defined and modeled. Because, if the real control is used, it is necessary to simulate the whole periphery and the Profibus connection as well as the whole plant by the physical model.

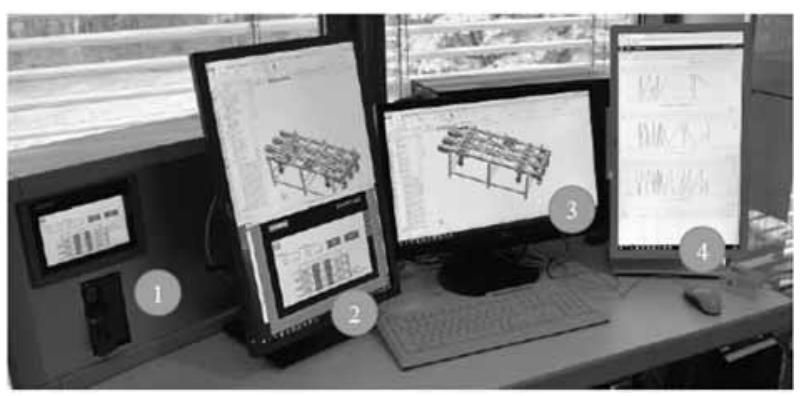

Figure 5: Illustration of Hil using a real controller with a human-machine interface (HMI) and a simulation unit (1) and the SII with a simulated controller, simulated HMI (2) and the multi-physical model (3) in the MCD, as well as exemplary cloud-based visualization of production data.

\section{Use Cases}

The demonstrated methodology of MBSE and a model environment for a co-simulation were implemented on the basis of two use cases in the field of teaching and research. The goals of the two cases are consistently different, but both are intended to contribute to the fact that product development can be carried out more cost-effectively, better and more efficiently in the future.

\subsection{Co-simulation for the Holistic Simulation of an Automated Production Plant}

The continuing, or rather successively increasing, scarcity of resources is now a worldwide problem, which is why the energy turnaround has been initiated. In the future, renewable resources such as photovoltaic systems or wind power plants are to be used in the context of manufacturing plants, and storage technologies (accumulators, capacitors) are to be integrated into the plants. Smart grids are being created in the manufacturing halls to meet the energy needs of manufacturing and to make optimal use of the distribution of resources. The energy supply must therefore be regulated via a further control system. 
However, the physical behavior of the accumulator or PV system cannot be represented in the common multiphysics simulation environments, nor can the extraction of energy from the utility grid. For this purpose, further simulations have to be used. In this case, MATLAB Simulink was used to simulate the accumulator. For the simulation of the process flow of the manufacturing plant, MCD was used. In the present simulation study, both environments were connected by means ofan OPC UA server. Furthermore, each tool has an OPC UA client. This is used to exchange state variables in discrete time steps and to test the energy management. For example, if an energy-intensive work step begins in the process simulation, the accumulator discharges to the maximum in its own simulation, as shown in Figure 6.

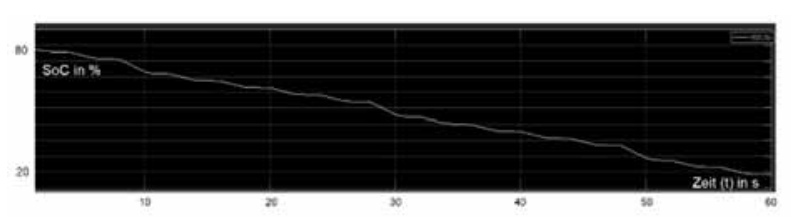

Figure 6: Course of the state of charge over the discharge process. (in MATLAB Simulink).

If this is completely discharged, the high-energy process is stopped and only restarted when the accumulator has been charged by the PV system.

This application primarily demonstrates the feasibility of co-simulation using OPC UA. However, all models are stored in the PLM system, because the structure of the product development process was carried out along the SE.

\subsection{Internship for Students as a Basis for Future Cooperation in Companies}

Another use case in regarding this topic was implemented in teaching. Every semester, students work in an interdisciplinary team on a development project based on a conveyor system on the chair's own I4.0 demonstrator.

In a fictitious development team, various roles are assigned whose task it is either to create and control the mechanical, electronic or information technology design, or to take care of the simulation accompanying the development, or to monitor the creation of the model already in the specification phase.

The business game should help to understand and comprehend the described basics of SE, because from the recording of the requirements from the customers' declarations to the commissioning of the conveyor belt, the students go through all the steps of the product development process, with all the necessary iterations.
By applying the methodology of SE already in the course of studies, a contribution is to be made to advance the introduction and application in industry.

\section{Summary and Outlook}

In summary, the approach of model-based development with the aid of PLM systems can meet the requirements placed on manufacturers of mechatronic systems by Industry 4.0. Simulation plays an important role here. Simulation in a multi-physics tool is not sufficient, so cosimulations must be used. One approach to this is to use the OPC UA standard. This approach can be extended so that it can be used to perform virtual commissioning. In order to establish this approach, it will be taught to students as close to practice as possible.

\section{References}

[1] Armin Barnitzke. Machine software must become more modular: Industry survey. In: Automationspraxis - die anwenderorientierte Fachzeitschrift für Führungskräfte in der Industrie; 2014 December: 12/2014. p. 1-3.

[2] Harashima F, Tomizuka M, Fukuda T. Mechatronics "What Is It, Why, and How?" An editorial. In: IEEE/ASME Transactions on Mechatronics 1996; 1: No. 1. p. 1-4.

[3] 2206. 2004. guideline VDI 2206. development methodology for mechatronic systems.

[4] Renault O, Expert P IM. Reuse/Variability Management and System Engineering. In: Poster Workshop of the Complex Systems Design \& Management Conference CSD\&M 2014; 2014.

[5] Kleiner S, Husung S, Schulze S O, Tschirner C, Kaffenberger R. Model Based Systems Engineering: Principles, Application, Examples, Experience and Benefits from a Practical Perspective. In: Systems Engineering Day 2016. p. 13-22.

[6] Eigner M, Roubanov D, Zafirov R. Model-based virtual product development. Springer; 2014.

[7] Fay Alexander. Continuous engineering of control systems (Conference on Continuous Plant Design 2013). Nuremberg, 20.03.2013

[8] Technical Operations International Council on Systems Engineering (INCOSE). Systems Engineering Vision (2025). URL https://www.incose.org/docs/defaultsource/aboutse/se-vision-2025.pdf?sfvrsn=b69eb4c6_4 Review date 02/22/2021.

[9] Cooper R G. Stage-gate systems: A new tool for managing new products. In: Business Horizons 1990; 33: No. 3. p. 44-54.

[10] Spath, D. Produktionsarbeit der Zukunft - Industrie 4.0: Studie. Stuttgart: Fraunhofer-Verl.; 2013.

SNE 31(4) - 12/2021 
[11] Russwurm S. Software: The Future of Industry. In: Sendler, U (Ed.): Industrie 4.0. Berlin, Heidelberg: Springer Berlin Heidelberg; 2013. p. 21-36.

[12] Szinovatz A, Müller C. Managing complexity in the innovation process From the stage-gate model to the survival-of-the-fittest model. In: Schoeneberg, K-P (Ed.): complexity management in companies. Wiesbaden: Springer Fachmedien Wiesbaden; 2014. p. 93-112.

[13] Geisberger E, Schmidt R. Final report of the project "ProMiS" - Project management for interdisciplinary system developments: From the topic area "Software in technical products - application of methods and procedures for engineering-based software development in production" within the framework of the BMBF research project "Research for the production of tomorrow" ; [Guide for the application of project management and system specification with a practical handbook on CD-ROM] ; [Software VDMA] ; sponsored by the Federal Ministry of Education and Research. Frankfurt am Main, c 2004 (Software vf177400).

[14] Augustin C. Guide to requirements analysis: software from the Methods and Procedures series. Frankfurt am Main: VDMA-Verl.

[15] Sauer O, Schleipen M, Ammermann C. Digital Factory Operation. In: Zülch, G; Stock, P (Eds.): Integration aspects of simulation: technology, organization and personnel. Karlsruhe: KIT Scientific; 2010. p. 559-566.

[16] VDI Guideline 3633; Sheet 1. 1993. VDI Guideline 3633, Sheet 1

[17] Feldmann K. Simulation-based planning systems for organization and production: model construction, simulation experiments, examples of use. Berlin [u.a.]: Springer; 2000.

[18] Reinhart G, Feldmann K, Heitmann K. Simulation - key technology of the future. In: status and perspectives. Munich: Utz, Wiss 1997.

[19] Lechler T, Fischer E, Metzner M, Mayr A, Franke J. Virtual Commissioning - Scientific review and exploratory use cases in advanced production systems. In: Procedia CIRP 2019; 81. p. 1125-1130.

[20] Siemens Industry Software GmbH \& Co. KG. : $N X$ : Transforming the entire product creation process through an integrated software solution for design, simulation and manufacturing. 2012
[21] Günther F C: Contribution to co-simulation in the overall system development of the motor vehicle. Munich, TU Munich. 2017

[22] Scheifele C: Platform for real-time co-simulation for virtual commissioning. Stuttgart, University of Stuttgart, Institute for Control Engineering of Machine Tools and Manufacturing Units: Dissertation. 2019

[23] Pritschow G, Berkemer T, Bürger T, Croon N, Korajda B, Röck S. The simulated machine tool. In: Heisel, U (ed.): Stuttgart impulses: shaping the future - setting the tone ; FtK 2003, Fertigungstechnisches Kolloquium Stuttgart, October 13-15, 2003 ; conference proceedings ; [written version of the lectures. Stuttgart: Ges. für Fertigungstechnik ; 2003. p. 219-246.

[24] Pritschow G, Röck S. "Hardware in the Loop" Simulation of Machine Tools. In: CIRP Annals 2004; 53: no. 1. p. 295-298.

[25] Schuh G. Innovationsmanagement: Handbuch Produktion und Management 3. 2., vollst. neu edit. und erw. ed. Berlin, Heidelberg: Springer; 2012.

[26] Eigner M, Stelzer R. Product Lifecycle Management: A Guide to Product Development and Life Cycle Management. 2nd, newly edited ed. Dordrecht: Springer; 2013.

[27] Eigner M, Roubanov D, Zafirov R. Model-based virtual product development. Berlin: Springer Vieweg; 2014.

[28] Sendler U. The PLM compendium: reference book of product lifecycle management. Springer Science \& Business Media; 2009.

[29] Lange J, Iwanitz F, Burke T J. OPC: From Data Access to Unified Architecture. 5th, revised edition. Berlin, Offenbach: VDE Verlag GmbH; 2014.

[30] Mahnke W, Leitner S-H, Damm M. OPC Unified Architecture. 1st ed. Berlin: Springer; 2009.

[31] VDI 4499. 2008. VDI 4499 Sheet 1 Digital Factory Basics

[32] WZL RWTHAachen. PLM. URL http://www.plminfo.de - Review date 08.06.2015

[33] Wenk M. Virtual Commissioning of Production Plants Effort-Benefits, Implementation Strategies, Future Developments. In: VDE-Verlag (Ed.) 2008 - Electrical Automation - Systems and Components. p. 533. 